\title{
Harmonic maps and isometric embeddings of the spacetime
}

\author{
S. Chervon ${ }^{a}$, F. Dahia ${ }^{b}$ and C. Romero ${ }^{a}$ \\ ${ }^{a}$ Departamento de Física, Universidade Federal da Paraíba, C. \\ Postal 5008, \\ 58051-970 João Pessoa, Pb, Brazil \\ ${ }^{b}$ Departamento de Física, Universidade Federal de Campina Grande, \\ 58109-970, Campina Grande, Pb, Brazil \\ E-mail: chervon@fisica.ufpb.br,fdahia@df.ufpb.brand cromero@fisica.ufpb.br
}

Typeset using REVTEX 


\begin{abstract}
We investigate harmonic maps in the context of isometric embeddings when the target space is Ricci-flat and has codimension one. With the help of the Campbell-Magaard theorem we show that any $n$-dimensional $(n \geqslant 3)$ Lorentzian manifold can be isometrically and harmonically embedded in a $(n+1)$-dimensional semi-Riemannian Ricci-flat space. We then extend our analysis to the case when the target space is an Einstein space. Finally, as an example, we work out the harmonic and isometric embedding of a Friedmann-Robertson-Walker spacetime in a five-dimensional Ricci-flat space and proceed to obtain a general scheme to minimally embed any vacuum solution of general relativity in Ricci-flat spaces with codimension one.
\end{abstract}

\title{
I. INTRODUCTION
}

Isometric embeddings of the spacetime in five dimensions have been considered with increasing interest in recent years. The development of the braneworld scenario by ArkaniHamed [1] et al and Randall \& Sundrum [2] has greatly favoured the idea among particle physicists and cosmologists that in lower energy regime our Universe may effectively be pictured as a four-dimensional hypersurface (brane) isometrically embedded in a fivedimensional manifold (bulk). The study of isometric embeddings has also been motivated by a proposal recently put forward and developed by Wesson and others [3], where vacuum $(4+1)$-dimensional field equations give rise to $(3+1)$-dimensional equations with source, the energy-momentum tensor of which being related to the extrinsic curvature of the spacetime [4]. Ultimately, all these ideas seems to have drawn inspiration from the old Kaluza-Klein theory [5].

Embedding theories are naturally subject to embedding theorems of differential geometry. Of interest here is a theorem due to Campbell and Magaard [6], which asserts that 
any n-dimensional Riemannian analytic manifold can be locally and isometrically embedded in a $(n+1)$-dimensional Riemannian analytic manifold, where the Ricci tensor of the latter vanishes. Campbell-Magaard's result may shed some light on our understanding of the mathematical structure of embedding theories, and applications of the theorem to the braneworld scenario, to five-dimensional non-compactified Kaluza-Klein gravity as well as to superstring theory have been found in recent years [8]. Extensions of the Campbell-Magaard theorem to more general cases have also been obtained $[9,10]$.

Due to the high interest aroused by spacetime embedding theories, different and specific types of embedding now start to be considered by theoretical physicists. Such is the case of the so-called ideal embeddings, in which the embedded space receives the "least amount of tension" from the surrounding (pseudo-Euclidean) space [11] An interesting kind of embedding that may have a connection with particle physics is provided by the so-called harmonic maps [12]. As models for physical theories, harmonic maps lead to nonlinear field equations, which in many respects bear a resemblance to Yang-Mills equations and to the Einstein equations for gravitation. Harmonic maps are also linked to the non-linear sigma models, first introduced by Schwinger, in the fifties, to describe massive, strongly interacting particles [13]. Recent developments in non-linear sigma models include interaction with gravity, inflationary cosmological models, Kaluza-Klein theories, among other topics [14].

Our aim here is to look at harmonic maps in the context of isometric embeddings and show that they represent a non-empty subclass of the class of all isometric embeddings when the target spaces are Ricci-flat with codimension one [15]. The paper is organized as follows. In section II we define the concept of isometric embedding of a n-dimensional semi-Riemannian manifold in the class of $(n+1)$-dimensional Ricci-flat semi-Riemannian manifolds. We then proceed to discuss the Campbell-Magaard embedding theorem. In Section III we briefly define harmonic maps, discuss how they are related to isometric embeddings and prove that for $n \geq 3$ any n-dimensional Lorentzian manifold can be isometrically and harmonically embedded in the class of $(n+1)$-dimensional Ricci-flat semi-Riemannian manifolds. In Section IV we give an example of a harmonic and isometric map between two 
semi-Riemannian manifolds in which the target space is a Ricci-flat manifold. An extension of these ideas to the case when the embedding manifold is an Einstein space is briefly treated in Section V. In Section VI we make some comments of geometric character on the meaning of harmonicity for isometric embeddings. We end with a remark on the role minimal embeddings could possibly play in the framework of embedding theories of the spacetime.

\section{ISOMETRIC EMBEDDINGS AND THE CAMPBELL-MAGAARD THEOREM}

The original version of the Campbell-Magaard theorem refers to Riemannian manifolds, i.e. those endowed with positive definite metrics. It can be shown, however, that this restrictive condition is not essential, so in what follows we shall consider semi-Riemaniann manifolds with metrics of indefinite signature. It is important to mention that throughout the paper embeddings and metrics are supposed to be analytic.

Let $\mathcal{M}_{\pi}^{n+1}$ denote the class of all $(n+1)$-dimensional manifolds $\bar{M}^{n+1}$ which share some geometrical characteristic $\pi$ (For example, $\pi$ may express a restriction of the following kind: to be Ricci-flat, to be an Einstein space, and so forth). A precise way of defining isometric embeddings of a n-dimensional manifold $M^{n}$ in the class of manifolds $\mathcal{M}_{\pi}^{n+1}$ is

Definition 1 We say that $M^{n}$ can be isometrically embedded in $\mathcal{M}_{\pi}^{n+1}$ if there exists at least one manifold $\bar{M}^{n+1} \in \mathcal{M}_{\pi}^{n+1}$ in which $M^{n}$ can be isometrically embedded.

Now, the Campbell-Magaard theorem states that any $M^{n}$ manifold can be isometrically embedded in the class $\mathcal{M}_{\pi}^{n+1}$ of vacuum (Ricci-flat) spacetimes. It is known that this theorem can be demonstrated by a method which consists in formulating the question as the initial value problem in general relativity. Let us briefly review this technique.

Consider the metric of the $(n+1)$-dimensional space written in a Gaussian coordinate system

$$
d s^{2}=\bar{g}_{i j}(x, \psi) d x^{i} d x^{j}+d \psi^{2}
$$


where $x=\left(x^{1}, \ldots, x^{n}\right)$, and Latin indices run from 1 to $n$ while Greek ones go from 1 to $n+1$.

We can verify that the Einstein vacuum equations $R_{\mu \nu}=0$ expressed in the above coordinates have the following structure [6]:

$$
\begin{aligned}
\frac{\partial^{2} \bar{g}_{i j}}{\partial \psi^{2}} & =F_{i j}\left(\bar{g}_{l m}, \frac{\partial \bar{g}_{l m}}{\partial \psi}\right) \\
\nabla_{j}\left(K^{i j}-g^{i j} K\right) & =0 \\
R+K^{2}-K_{i j} K^{i j} & =0,
\end{aligned}
$$

where $F_{i j}$ are analytical functions of their arguments, $\nabla_{j}$ is the covariant derivative with respect to the induced metric $g_{i k}=\bar{g}_{i j}(x, \psi=$ const $) ; K=g^{i j} K_{i j}, R$ and $K_{i j}$ denote, respectively, the scalar curvature and the extrinsic curvature of the hypersurface $\psi=$ const. Recall that in the Gaussian coordinates of (1) the extrinsic curvature assumes the simple form:

$$
K_{i j}=-\frac{1}{2} \frac{\partial \bar{g}_{i j}}{\partial \psi} .
$$

Owing to the Bianchi identities not all of the above set of equations are independent. In fact, the second and third equations need to be imposed only on some particular hypersurface, since they are propagated by the first one [6]. These equations will be referred to as constraint equations.

To fix ideas let us chose, in the foliation defined above, a particular hypersurface, say, $\psi=0$. According to the Cauchy-Kowalewskaya theorem [7], equation (2) always has a unique analytical solution $\bar{g}_{i j}(x, \psi)$ provided that the following analytical initial conditions are specified:

$$
\begin{aligned}
& \bar{g}_{i j}(x, 0)=g_{i j}(x) \\
& \left.\frac{\partial \bar{g}_{i j}}{\partial \psi}\right|_{\psi=0}=-2 K_{i j}(x) .
\end{aligned}
$$

From the perspective of the embedding problem these initial conditions represent, respectively, the metric and the extrinsic curvature of the hypersurface $\psi=0$, whereas the 
solution of equation (2) gives the metric of the $(n+1)$-dimensional space. Thus, if we can guarantee that, for any given metric $g_{i k}(x)$, the constraint equations always admit a solution, then the theorem is proved, since the solution found $\bar{g}_{i j}(x, \psi)$ will satisfy $R_{\mu \nu}=0$. Clearly, the embedding map is then given by the equation $\psi=0$.

It turns out, as Magaard has proved [6], that the constraint equations always have a solution. Indeed, by simple counting operation we can see that there are $n(n+1) / 2$ unknown functions (the independent elements of extrinsic curvature) and $n+1$ constraint equations, since the metric $g_{i j}(x)$ must be considered as a given datum. For $n \geq 3$, the number of variables is greater than the number of equations. Thus using equation (4) to express one element of $K_{i j}$ in terms of the others, Magaard has shown that equation (3) can be put in a canonical form with respect to $n$ components of $K_{i j}$ conveniently chosen. Then, once more, the Cauchy-Kowalewskaya theorem ensures the existence of the solution. It is important to note that, as we have said before, the number of variables is greater than the number of equations; in this sense, we can say that there are $(n+1)(n / 2-1)$ degrees of freedom left over.

From this analysis we see that the embedding space is may not be unique. Different choices of the free elements of $K_{i j}$ may lead to different embedding spaces. Now let $\mathcal{S}_{\pi}^{n+1}$ be the set of all possible embedding spaces for $M^{n}$ constructed by employing the CampbellMagaard method. Of course $\mathcal{S}_{\pi}^{n+1} \subset \mathcal{M}_{\pi}^{n+1}$. At this point, we wonder if, among all the embeddings of $M^{n}$ in $\mathcal{S}_{\pi}^{n+1}$, there exists at least one that constitutes a harmonic map. In the next section we define harmonic embeddings in $\mathcal{M}_{\pi}^{n+1}$.

\section{HARMONIC MAPS}

Definition 2 We say that $M^{n}$ can be harmonically embedded in $\mathcal{M}_{\pi}^{n+1}$ if there exists at least one manifold $\bar{M}^{n+1} \in \mathcal{M}_{\pi}^{n+1}$ in which $M^{n}$ can be harmonically embedded, i.e., there exists a map $\phi: M^{n} \rightarrow \bar{M}^{n+1}$ that extremizes the action: 


$$
I[\phi]=\int d^{n} x \sqrt{|g|} g^{i j} \phi_{i}^{\mu} \phi_{j}^{\nu} H_{\mu \nu}
$$

where $H_{\mu \nu}$ represents the metric tensor of $\bar{M}^{n+1}$ and we are using the notation $\phi_{i}^{\mu}=\frac{\partial \phi^{\mu}}{\partial x^{i}}$.

Of course in order for $\phi$ to be also an isometric embedding in $\mathcal{M}_{\pi}^{n+1}$ we must have $\bar{M}^{n+1} \in \mathcal{S}_{\pi}^{n+1}$ ( here the property $\pi$ means that $H_{\mu \nu}$ is the metric of a vacuum space). Assuming that $\bar{M}^{n+1} \in \mathcal{S}_{\pi}^{n+1}$, then, as we know, there exist coordinates of $\bar{M}^{n+1}$ adapted to the embedding, i.e., we can put the metric $H_{\mu \nu}$ in the Gaussian form

$$
d s^{2}=H_{i j}(x, \psi) d x^{i} d x^{j}+d \psi^{2},
$$

with

$$
H_{i j}(x, 0)=g_{i j}(x) .
$$

In these coordinates we have the following relations:

$$
\begin{gathered}
\phi^{i}=x^{i}, \\
\phi^{n+1}=\psi(x) .
\end{gathered}
$$

Thus, the effective action becomes

$$
I[\psi]=\int d^{n} x \sqrt{|g|} g^{i j}\left(H_{i j}(x, \psi)+\psi_{i} \psi_{j}\right)
$$

and the extremization condition

$$
\frac{\delta I}{\delta \psi}=0
$$

gives

$$
\frac{1}{\sqrt{|g|}} \partial_{i}\left(\sqrt{|g|} g^{i j} \psi_{j}\right)-\frac{1}{2} g^{i j} \partial_{\psi} H_{i j}(x, \psi)=0
$$

From this equation we can conclude that the isometric embedding $\psi=0$ is a harmonic map if and only if 


$$
\left.\frac{1}{2} g^{i j} \partial_{\psi} H_{i j}(x, \psi)\right|_{\psi=0}=0
$$

which, taking (5) into account, is equivalent to

$$
K=0 .
$$

Therefore the existence of the embedding space $\bar{M}^{n+1}$ depends on the existence of a solution of the constraint equations (3), (4) and (17). In fact, provided that these equations are satisfied for some $K_{i j}$ and $g_{i j}$, the particular solution of (2) with these $K_{i j}$ and $g_{i j}$ as initial data, represents, in coordinates (1), a vacuum metric in $n+1$ dimensions. In this case, the map $\psi=0$ will be an isometric and harmonic embedding. Thus, we have proved the following:

A manifold $M^{n}$ can be isometrically and harmonically embedded in $\mathcal{M}_{\pi}^{n+1}$, if and only if there exist functions $K_{i j}$ which satisfy the equations

$$
\begin{gathered}
\nabla_{j}\left(K^{i j}-g^{i j} K\right)=0 \\
R+K^{2}-K_{i j} K^{i j}=0, \\
K=0 .
\end{gathered}
$$

At this point it would be nice if we were able to prove that it is always possible to choose the extrinsic curvature tensor in such a manner that its components satisfy the constraint equations, i.e, that the equations are not incompatible. It turns out that, this is the case, at least for Lorentzian manifolds and $n \geqslant 3$, as it is shown in the Appendix. Therefore, the following proposition holds true:

Any analytical Lorentzian manifold $M^{n}(n \geq 3)$ can be analytically, isometrically and harmonically embedded in the class of $(n+1)$ - dimensional vacuum spaces. 


\section{A SIMPLE EXAMPLE}

As a simple application of the ideas discussed above let us consider the class of all $n$ dimensional manifolds whose scalar curvature $R$ vanishes. Then, an obvious choice for the initial data required by the Campbell-Magaard theorem is to take the extrisinc curvature

tensor $K_{i j}=0$. In this case, the constraint equations are trivially satisfied. Therefore, we conclude that a sufficient condition for a $n$-dimensional manifold with vanishing curvature scalar $R$ to be isometrically and harmonically embedded in a Ricci-flat $(n+1)$ dimensional manifold is that $K_{i j}=0$. This is the case, for instance, of the embedding of

the 4-dimensional Friedmann-Robertson-Walker metric

$$
d s^{2}=d t^{2}-t\left(d x^{2}+d y^{2}+d z^{2}\right)
$$

in the 5-dimensional Ricci-flat space

$$
d s^{2}=d t^{2}-t\left(d x^{2}+d y^{2}+d z^{2}\right)-d \psi^{2}
$$

the embedding taking place for $\psi=0$.

This example may be easily generalized. Let

$$
d s^{2}=g_{i j}(x) d x^{i} d x^{j}
$$

be the metric of $n$-dimensional space with $R=0$. It is then straightforward to verify that this space can be isometrically and harmonically embedded in the $(n+1)$-dimensional Ricci-flat space

$$
d s^{2}=f(\psi) g_{i j}(x) d x^{i} d x^{j}-d \psi^{2}
$$

where $f$ is a differentiable function with $f(0)=1$ and $f^{\prime}(0)=0$. Indeed, in this case the extrinsic curvature tensor is given by

$$
K_{i j}=\frac{-f^{\prime}(0) g_{i j}(x)}{2}=0
$$


hence all constraint equations are satisfied. Since Ricci-flat spaces have zero scalar curvature,

we can state, as a corollary of the above, that any $n$-dimensional vacuum solution of general relativity may be isometrically and harmonically embedded in a $(n+1)$-dimensional Ricci-flat space.

\section{HARMONIC MAPS AND EINSTEIN SPACES}

All we have done up to this point may easily be carried over into more general settings. Consider, for example, an extended version of the Campbell-Magaard theorem [9], which states that any $n$-dimensional semi-Riemannian manifold can be locally embedded in a $(n+1)$-dimensional Einstein space. In this case the Einstein equations $G_{\mu \nu}=\Lambda g_{\mu \nu}$ are equivalent to the set

$$
\begin{gathered}
\frac{\partial^{2} \bar{g}_{i j}}{\partial \psi^{2}}=F_{i j}\left(\bar{g}_{l m}, \frac{\partial g_{l m}}{\partial \psi}, \Lambda\right) \\
\nabla_{j}\left(K^{i j}-g^{i j} K\right)=0 \\
R+K^{2}-K_{i j} K^{i j}=-2 \Lambda
\end{gathered}
$$

where $\Lambda$ is a constant (usually referred to as the cosmological constant of the embedding space). The extension of the previous theorem to embeddings of n-dimensional $(n \geqslant 3)$ Lorentzian manifolds in Einstein spaces is readily seen to hold ( see Appendix ). Thus, we have the following result:

Let $M_{\Lambda}^{n+1}$ be the class of the $(n+1)-$ dimensional Einstein spaces with constant $\Lambda$. Then, any analytical Lorentzian manifold $M^{n}(n \geq 3)$ can be analitically, isometrically and harmonically embedded in $M_{\Lambda}^{n+1}$.

\section{FINAL REMARKS}


In section III we have generically defined harmonic maps as those maps which extremizes the action $I[\phi]=\int d^{n} x \sqrt{|g|} g^{i j} \phi_{i}^{\mu} \phi_{j}^{\nu} H_{\mu \nu}$. Now if, in addition, the embedding is assumed to be isometric, we have

$$
\phi_{i}^{\mu} \phi_{j}^{\nu} H_{\mu \nu}=g_{i j}
$$

In this case the action may be rewritten as

$$
I[\phi]=\int d^{n} x \sqrt{|g|} g^{i j} g_{i j}=n \int d^{n} x \sqrt{|g|}
$$

Therefore we see that harmonic embeddings extremize the volume in the induced metric $g_{i j}$. Let us make this statement more precise. If $M^{n}$ is isometrically and harmonically embedded in $\bar{M}^{n+1}$, and if $D$ is a domain of $M^{n}$ with a regular boundary $\partial D$, then the volume of $D$ in the induced metric is less ( greater, depending on the signature of $g_{i j}$ ) than or equal to the volume of any other submanifold of $\bar{M}^{n+1}$ with the same boundary. Embeddings which satisfy this property are referred to, in the mathematical literature, as minimal [16]. ( In fact, for Riemannian spaces it is known that an isometric embedding is minimal if and only if it is a harmonic map [17].) Let us just mention that an important characterization of a minimally embedded space $M^{n}$ lies in the fact that its mean curvature vector $H$ vanishes for all points $p \in M^{n}$. In fact it is a well-known fact that $H$ vanishes if and only if the trace of the extrinsic curvature $K_{i j}$ of the hypersurface $\psi=0$ is zero.

Finally, we would like to raise the question whether minimal embeddings may play a role in higher-dimensional theories of the spacetime. In a paper published some years ago Mc Manus [18] posed a couple of questions concerning the so-called induced matter models. In one of the questions it was asked whether any energy-momentum tensor could be obtained by an embedding mechanism. Due to the Campbell-Magaard theorem we now know that the answer is yes, since any solution to the Einstein field equations corresponding to any arbitrary energy-momentum tensor may be locally embedded in a five-dimensional Ricci-flat space. The second question, perhaps more relevant, was if there were a valid mechanism for determining the choice of the four-dimensional hypersurfaces as the ones which would 
be physically observables. We know that in the induced matter scheme the dynamics of the surrounding space (bulk) is governed by the Einstein field equations in vacuum. However, the choice of the foliations of the bulk by four-dimensional hypersurfaces is entirely arbitrary. By requiring these foliations to obey some (yet unknown) fundamental physical principle, perhaps some progress could be made by linking the dynamics of the spacetime to the dynamics of the bulk. In the specific case of minimal embeddings one has a variational principle, namely, the extremization of the action defined by the equation (18), i.e. the extremization of the volume of the spacetime. This, at least, would put some constraints in the possible choices of the foliation of the hypersurfaces. We hope these considerations may stimulate work in this direction.

\section{ACKNOWLEDGEMENTS}

S.Chervon thanks the Physics Department of Universidade Federal da Paraiba at Joao Pessoa (Brazil) for warm hospitality and CAPES for financial support. C. Romero thanks $\mathrm{CNPq}$ for grant and financial support.

\section{APPENDIX}

Our aim in this appendix is to prove that, for $n \geq 3$, the constraint equations of the section III can always be satisfied by an appropriate choice of the extrinsic curvature tensor. Considering the possibility of further applications to general relativity let us turn our attention to the case when $M^{n}$ is Lorentzian.

For the sake of simplicity let us consider a Gaussian coordinate system of the manifold $M^{n}$, where $x^{1}$ is a timelike coordinate. In these coordinates the metric $g_{i j}$ assumes the following form

$$
d s^{2}=-\left(d x^{1}\right)^{2}+g_{A B}(x) d x^{A} d x^{B},
$$


where $A$ and $B$ run from 2 to $n$.

Of course, $g_{A B}(x)$ is positive definite ( it is the induced metric on the submanifold $x^{1}=$ const), hence the diagonal components of $g_{A B}$ are all positive quantities. Thus, in terms of this coordinate system the constraint equation (3) reads

$$
\frac{\partial K^{1 i}}{\partial x^{1}}+\frac{\partial K^{A i}}{\partial x^{A}}-\Gamma_{j m}^{i} K^{j m}-\Gamma_{j m}^{j} K^{i m}=0
$$

where $\Gamma_{j m}^{i}$ are the Christoffell symbols calculated with the metric $g_{i j}$. As we emphasized previously for $n \geq 3$ there are more components $K^{i j}$ than equations. On the other hand, since there are $n+2$ constraint equations we can choose $n+2$ components of $K^{i j}$ to be treated as unknown functions in order to satisfy the constraint equations. Let us select $K^{11}, K^{12}, \ldots, K^{1 n}, K^{22}$ and $K^{33}$. The remaining components can be considered as independent functions, so they be taken equal to zero.

After some algebra the constraints equations (4) and (17) may be put in the following form:

$$
\begin{aligned}
-K^{11}+g_{22} K^{22}+g_{33} K^{33} & =0 \\
\left(K^{11}\right)^{2}-g_{A B} K^{1 A} K^{1 B}-\operatorname{det} g_{[2,3]} K^{22} K^{33} & =\frac{R}{2},
\end{aligned}
$$

where det $g_{[2,3]}=g_{22} g_{33}-\left(g_{23}\right)^{2}$ corresponds to the area measure of the surface spanned by the coordinates $x^{2}$ and $x^{3}$. Evidently det $g_{[2,3]}>0$, since a positive definite metric satisfies the Cauchy-Schwarz inequality: $\left\|X_{1}\right\|^{2}\left\|X_{2}\right\|^{2} \geq\left(X_{1}, X_{2}\right)^{2}$, where $X_{i}=\frac{\partial}{\partial x^{i}}$.

From (20) it follows that

$$
K^{33}=\frac{K^{11}-g_{22} K^{22}}{g_{33}}
$$

Substituting the above equation into (21) we find a quadratic equation for $K^{22}$ :

$$
\left(\frac{g_{22}}{g_{33}} \operatorname{det} g_{[2,3]}\right)\left(K^{22}\right)^{2}-\left(K^{11} \frac{\operatorname{det} g_{[2,3]}}{g_{33}}\right) K^{22}+\left(\left(K^{11}\right)^{2}-g_{A B} K^{1 A} K^{1 B}-\frac{1}{2} R\right)=0 .
$$

Now, this equation can be solved for $K^{22}$ if the discriminant is non-negative. Clearly, in the case of a positive discriminant $K_{22}$ can be written as an analytical function of $K^{1 i}$ $(i=1, \ldots, n)$ and the same holds for $K^{33}$. 
Surely, using the above relations we substitute the components $K^{22}$ and $K^{33}$ into (19) obtain a first-order partial differential equation for $K^{1 i}$, which can be put in the following form

$$
\frac{\partial K^{1 i}}{\partial x^{1}}=F^{i}\left(K^{1 j}, \frac{\partial K^{1 j}}{\partial x^{A}}\right)
$$

Then, from the Cauchy-Kowalewskaya theorem, we conclude that if $F^{i}$ is analytical in its arguments at some point, the origin, for example, then there exists a unique analytical solution satisfying the initial condition

$$
K^{1 i}\left(x^{1}=0, x^{2}, \ldots, x^{n}\right)=W^{i}\left(x^{2}, \ldots, x^{n}\right)
$$

where $W^{i}$ are arbitrary analytical functions in the origin.

In order for $F^{i}$ to be analytic at the origin, it is sufficient that the discriminant of the quadratic equation for $K^{22}$ be positive at this point. In other words, we must have

$$
\left(W^{1} \frac{\operatorname{det} g_{[2,3]}}{g_{33}}\right)^{2}-4\left(\frac{g_{22}}{g_{33}} \operatorname{det} g_{[2,3]}\right)\left(\left(W^{1}\right)^{2}-g_{A B} W^{A} W^{B}-\frac{1}{2} R\right)>0
$$

It is not difficult to verify that it is always possible to have the above inequality satisfied. Indeed, with the choice $W^{1}=0$ and $g_{A B} W^{A} W^{B}>-\frac{1}{2} R$, for instance, it is evident that (26) holds. Therefore, we conclude that any Lorentzian spacetime can be minimally and analytically embedded in the class of five-dimensional vacuum spaces.

This result can be trivially extended to the case of the embedding in the class of Einstein spaces with a certain specified $\Lambda$. As far as the constraint equations are concerned we have just to add the constant $\Lambda$ on the right hand side of equation (21). Then, following the same procedure outlined above the existence of solutions of the constraint equations is guaranteed just by requiring that $W^{1}=0$ and $g_{A B} W^{A} W^{B}>-\frac{1}{2} R-\Lambda$. 


\section{REFERENCES}

[1] N. Arkani-Hamed, S. Dimopoulos, and G. R. Dvali, Phys. Lett. B429, 263 (1998), hep-ph/9803315. N. Arkani-Hamed, S. Dimopoulos, and G. R. Dvali, Phys. Rev. D59, 086004 (1999), hep-ph/9807344. I. Antoniadis, N. Arkani-Hamed, S. Dimopoulos, and G. R. Dvali, Phys. Lett. B436, 257 (1998), hep-ph/9804398

[2] L. Randall and R. Sundrum, Phys. Rev. Lett. 83, 3370, hep-ph/9905221. L. Randall and R. Sundrum, Phys. Rev. Lett. 83, 3370, 4690, hep-th/9906064.

[3] J. M. Overduin and P. S. Wesson, Phys. Reports 283, 303 (1997). P. S. Wesson, SpaceTime-Matter (World Scientific, Singapore, 1999)

[4] M. D. Maia, gr-qc/9512002

[5] T. Kaluza, Sitzungsber. Preuss. Akad. Wiss. Berlin (Math. Phys.) K1 966 (1921). O. Klein, Z. Phys. 37895

[6] J. Campbell, A Course of Differential Geometry (Oxford: Claredon, 1926). L. Magaard, Zur einbettung riemannscher Raume in Einstein-Raume und konformeuclidische Raume (PhD Thesis, Kiel, 1963).

[7] See, for instance, R. Courant and D. Hilbert, Methods of Mathematical Physics, vol. II ( Joh Wiley \& Sons, 1989).

[8] C. Romero, R. Tavakol, and R. Zalaletdinov, Gen. Rel. Grav. 28, 365 (1996). J. E. Lidsey, R. Tavakol and C. Romero, Mod. Phys. Lett. A12, 2319 (1997). S. S. Seahra and P. S. Wesson, Class. Quant. Grav. 20, 1321 (2003). F. Dahia and C. Romero, grqc/0308056. J. E. Lidsey, C. Romero, R. Tavakol and S. Rippl, Class. Quant. Grav. 14, 865 (1997). J. E. Lidsey, R. Tavakol and C. Romero, Mod. Phys. Lett. A12, 2319 (1997). J. E. Lidsey, Phys. Lett. B 417 (1998).

[9] F. Dahia and C. Romero, J. Math. Phys., 43, 6, 3097 (2002). 
[10] F. Dahia and C. Romero, J. Math. Phys., 43, 11, 5804, (2002). E. Anderson F. Dahia, J. Lidsey and C. Romero, J. Math. Phys. 44, 5108, gr-qc/0111094. E. Anderson and J. Lidsey, Class. Quant. Grav. 18, 4831 (2001)

[11] S. Haesen and L. Verstraelen, gr-qc/0308066

[12] F. B. Fuller, Proc. Natl. Acad. Sci. 40987 (1954). J. Eells and J. H. Sampson, American Journal of Mathematics 86, 109 (1964). J. Eels and L. Lemaire, Bull. London Math. Soc. 101 (1968). C. W. Misner, Phys. Rev. D 184510 (1978).

[13] J. Schwinger, Ann. Phys. 2, 407 (1957). See also T. H. Skyrme, Proc. Roy. Soc. A247, N. 1249, 260 (1958) and M. Gell-Mann, M. Levy, Nuovo Cim. 26, N.4, 705 (1960).

[14] V. De'Alfaro, S. Fubini, G. Furlan, Nuovo Cim. A 50 No 4523 (1979). P. Tataru-Mihai , Nuovo.Cim. A 51 No 2169 (1979). G. Ghika, M. Visinescu, Nuovo Cim. B 59 No 159 (1980). G. Ivanov. Teor. i Mat.Fiz. 57 N 145 (1983). S. V. Chervon, Izv. Vys. Uch. Zav. Fisika N 8, 89 (1983). A. M. Perelomov, Phys.Reports 146 No 3136 (1987). C. Lechner, S. Husa, P. C. Aichelburg, Phys.Rev. D62(2000) 044047. S. V. Chervon, J Astroph. Astron. 16, Suppl. 65 (1995). S. V. Chervon, Int.J.Mod.Phys. A 17, 4451 (2002).

[15] S. Chervon and C. Romero, Harmonic maps as a subclass of isometric embeddings of the spacetime in five dimensions, Proceedings of the Third International School-Seminar ( Problems of Theoretical and Observational Cosmology), Ulyanovsk, Russia (to be published).

[16] M. P. do Carmo, Riemannian Geometry (Birkhäuser, Boston, 1992) M. Dajczer, Submanifolds and Isometric Immersions (Publish or Perish, Houston, 1990)

[17] J. Eells and J.Sampson, Am. J. Math. 86 (1964) 109-169. S. Montaldo, Minimality and harmonicity in differential geometry, Pubblicazioni interne, Gruppo di Geometria Riemanniana, Università di Cagliari, Italy. 
[18] D. J. Mc Manus, J. Math. Phys. 35, 4889 (1994). 